\title{
Intra-bone marrow transplantation confers superior multi-lineage \\ engraftment of murine aorta-gonad mesonephros cells over intravenous \\ transplantation
}

\author{
Alejandra Sanjuan-Pla1 , Damia Romero-Moya ${ }^{1}$, Cristina Prieto ${ }^{1}$ \\ Clara Bueno ${ }^{1}$ Anna Bigas², Pablo Menendez ${ }^{1,3, *}$
}

\begin{abstract}
1Josep Carreras Leukemia Research Institute and School of Medicine. University of Barcelona. Barcelona. Spain. ${ }^{2}$ Program in Cancer Research, Institut Hospital del Mar d'Investigacions Mèdiques (IMIM), Parc de Recerca Biomèdica de Barcelona. Barcelona. Spain. 3Institució Catalana de Recerca i Estudis Avançats (ICREA). Barcelona. Spain.
\end{abstract}

Running Title: Intra-bone marrow transplantation of AGM HSPCs.

Key words: E11.5 AGM, intra-bone marrow transplantation, intravenous injection, embryonic hematopoiesis.

"Correspondence should be addressed to:

Alejandra Sanjuan-Pla \& Pablo Menendez.

Josep Carreras Leukemia Research Institute.

School of Medicine. University of Barcelona.

Casanova 143, 08036. Barcelona. Spain.

asanjuan@carrerasresearch.org ; pmenendez@carrerasresearch.org 


\begin{abstract}
Hematopoietic stem cell (HSC) engraftment has been achieved using single cell transplantation of prospectively highly purified adult HSC populations. However, bulk transplants are still performed when assessing the HSC potential of early embryonic hematopoietic tissues such as the aorta-gonad mesonephros (AGM) due to very low HSC activity content early in development. Intra-bone marrow transplantation (IBMT) has emerged as a superior administration route over intravenous (IV) transplantation for assessing the reconstituting ability of human HSCs in the xenotransplant setting since it bypasses the requirement for homing to the bone marrow (BM). Here we compared the ability of IBMT and IV administration of E11.5 AGM-derived cells to reconstitute the hematopoietic system of myeloablated recipients. IBMT resulted in higher levels of AGM HSC long-term multilineage engraftment in the peripheral blood, BM, spleen and thymus of primary and secondary recipients, and in limiting dilution experiments. The administration route did not skew the multilineage contribution pattern but IBMT conferred higher Lin-c-kit+Sca-1+ (LSK) long-term engraftment, in line with the superior IBMT reconstitution. Therefore, IBMT represents a superior administration route to detect HSC activity from developmentally early sources with limited HSC activity content, such as the AGM.
\end{abstract}




\section{INTRODUCTION}

Hematopoiesis allows the formation of blood cells from a hematopoietic stem cell (HSC) through a stepwise process, and occurs at different sites through ontogeny in mice and humans [1]. During mouse embryogenesis, definitive HSCs capable of reconstituting adult irradiated recipients are generated at embryonic day (E) 10.5-E11.5 from the ventral wall of the dorsal aorta-gonad-mesonephros (AGM) [2] , from the umbilical vein [3] and from the placenta [4]. Due to the constant migration of HSCs through blood circulation during development, an incomplete picture remains regarding which locations constitute de novo HSC production sites and which are pre-HSC-to-HSC maturation sites. At approximately E12.5, HSCs colonize the fetal liver (FL) to further migrate to the bone marrow (BM) where they reside for the lifespan of the organism [1].

In the murine and human settings, the transplantation assay is the in vivo gold standard test to study HSC function. HSC transplantation is widely employed in experimental and clinical hematology, and refinement of HSC transplantation assays have resulted in improved HSC engraftment/read-out. For instance, murine HSC transplantation is now optimized to be performed at the single cell level, and has been crucial in revealing the functional heterogeneity of murine and human HSC compartments. In the mouse, HSC transplantation assays are mainly performed by intravenous (IV) administration due to the intrinsic circulatory properties of hematopoietic cells. Successful engraftment then requires blood circulation and migration of HSCs into the BM, which offers a supportive niche. Alternatively, direct injection of cells within the BM has been successfully employed to engraft human HSCs [5-8], leukemia cells $[9,10]$ and mesenchymal sarcoma cells $[11,12]$ in immunodeficient mice. Intra-BM transplantation (IBMT) has shed light on the homing and repopulation properties of human HSCs, and has been shown to result in higher levels of multilineage long-term reconstitution than IV injection. IBMT also 
allows detection of HSC potential from non-hematopoietic tissues, such as liver, muscle, and human pluripotent stem cells (hPSCs). Interestingly, IBMT has also been employed with whole murine BM cells $[13,14]$; however, in one study IBMT did not result in an enhanced engraftment compared with the IV technique [15].

Recently, the group of Daley tested the impact of HSC ontogeny (embryonic vs adult) and the recipient's age (newborn or adult) on the engraftment potential upon transplantation [16]. They showed that embryonic HSCs from E9.5-10.5 preferentially engrafted neonatal mice. Despite the wide use of IBMT in the HSC xenotransplantation setting, no studies so far have assessed the engraftment ability of murine hematopoietic cells of embryonic origin by these different administration routes. We hypothesized that IBMT could represent a superior strategy to detect/read-out hematopoietic reconstitution of embryonic cells given their scarcity at early stages of development. Indeed, limiting dilution assays have reported that an AGM at E11.5 contains approximately one definitive HSC [17]. In this study, we have compared the ability of IV and IBMT administration of E11.5 AGM cells to reconstitute the hematopoiesis in a syngeneic transplantation setting. Our data shows that IBMT confers superior multi-lineage engraftment of murine AGM cells over IV transplantation, thus offering an improved read-out to study early developmental hematopoiesis function. 


\section{MATERIAL AND METHODS}

\section{Mice and embryo dissection}

C57BL/6J (CD45.2) and B6/SJL (CD45.1) mice were obtained from Charles River Laboratories. Both strains were housed and maintained at the Animal Facility of the Barcelona Biomedical Research Park (PRBB) following the recommendations of the Federation of European Laboratory Animal Science Associations. Timed matings were set up late in the evening and vaginal plugs were checked in the early morning. The day-of positive plug detection was considered E0.5. At E11.5, pregnant C57/BL6 females were sacrificed by cervical dislocation. The uterus was collected in PBS and embryo dissection was performed in PBS+10\% FCS under a stereomicroscope. AGMs were dissected from each embryo, pooled and incubated for $30 \mathrm{~min}$ at $37^{\circ} \mathrm{C}$ in PBS $+10 \%$ FCS with $400 \mu \mathrm{g} / \mathrm{ml}$ collagenase type I. The cells were then washed four times with PBS+10\% FCS before transplantation. All experimental procedures were approved by PRBB and Generalitat de Catalunya animal ethics committee.

\section{Transplantation}

Prior to transplantation, IBMT recipients were analgesized subcutaneously $(2 \mathrm{mg} / \mathrm{kg}$ meloxicam and $0.1 \mathrm{mg} / \mathrm{kg}$ buprenorfina) and anesthesized by $4 \%$ isofluorane inhalation. For IBMT injections, the femur-tibia joint was perforated with a $25 \mathrm{G}$ needle and subsequently cells were injected into the retrieved orifice with a $27 \mathrm{G}$ needle in a volume of $25 \mu \mathrm{L}[5,18]$. IV injections were performed in the lateral tail vein in a volume of $200 \mu \mathrm{L}$ [5]. One fresh AGM embryo equivalent (ee) CD45.2 donor cells were either IV or IBMT injected along with 400,000 unfractionated adult BM cells (CD45.1) into lethally irradiated (9 Gy) CD45.1 mice ( $n=12$ IV, $n=10$ IBMT from $n=4$ independent experiments). Transplanted animals received antibiotics in drinking water for 4 weeks after transplantation. Donor chimerism in peripheral blood (PB) was assessed at 3, 8, 12 (data not 
shown) and 16 weeks post-transplantation. Mice with $\geq 1 \%$ CD45.2+ cells in PB and multilineage (myeloid, B and T-cell) reconstitution at 16 weeks post-transplantation were scored as reconstituted and were considered for further analysis. Recipients were sacrificed at week 18 after transplantation for tissue analysis. For secondary transplantation, unfractionated BM cells $\left(6 \times 10^{6}\right)$ from IV- and IBMT-engrafted primary recipients were IV injected as described in lethally irradiated secondary CD45.1 mice ( $n=2$ secondary recipients per primary recipient; total $n=12$ ). CD45.2+ donor engraftment was assessed at 3, 9, 16 and 20 weeks post-transplantation. As an AGM limiting cell dose approach, 1 and 0.5 AGM ee CD45.2+ cells were IV or IBMT cotransplanted with 20,000 adult unfractionated BM CD45.1+ support cells into lethally irradiated CD45.1 mice. Mice with $\geq 1 \%$ CD45.2+ cells were considered donor-engrafted. A total of 10 recipients were transplanted for each group in $n=5$ independent experiments.

\section{Donor chimerism analysis in PB and tissues}

PB was collected from the facial vein directly into EDTA tubes. Red blood cell (RBC) lysis was performed using QuickLysis (Cytognos, Salamanca, Spain). Cells were then stained in PBS $+5 \%$ FCS with fluorochrome-conjugated antibodies. CD45.1-APC and CD45.2-APC-Cy7 were used for allotype discrimination in transplanted mice. In multi-lineage analysis, cells were stained with Mac-1-V450, B220-PE-Cy7 and CD4/CD8-PE for myeloid, B-cell and T-cell analysis, respectively. Cells were analyzed using a FACS Canto II cytometer (BD Bioscience) and FlowJo software (Tree Star).

For BM analysis, femurs and tibiae were harvested and pooled from IV recipients. For IBMT recipients, injected tibiae (IT) were harvested separately from the remaining femur and from bones of the contralateral limb. A BM cell suspension was obtained by crushing the bones and 
$\mathrm{RBC}$ lysis was performed before staining. For Lineage-Sca- $1^{+} \mathrm{c}-\mathrm{kit} \mathrm{t}^{+}(\mathrm{LSK})$ staining, cells were incubated with the following antibodies: lineage cocktail (comprising Mac1, Gr-1, B220, CD4, CD8a, CD5, Ter119, all directly conjugated to PE-Cy5), c-kit-PE, Sca-1-FITC, CD45.1-APC and CD45.2-APC-Cy7. For spleen and thymus analysis, single cell suspensions were obtained by dissociating the tissue using a $70 \mu \mathrm{m}$ cell strainer and a syringe plunger. After RBC lysis, splenic cells were stained with B220-VioBlue, CD8-FITC, CD4-PE, CD45.1-APC and CD45.2-APC-Cy7, whereas thymus cells were stained with CD8-FITC, CD4-PE, CD45.1-APC and CD45.2-APCCy7. Further details on FACS antibodies used are shown in Table S1.

\section{Statistical Analysis}

Data are expressed as mean \pm s.e.m of independent experiments. Statistical comparisons were performed using either paired or unpaired Student's $t$ test as appropriate. Statistical significance was defined as $P$-value<0.05. Kaplan-Meier plots representing donor engraftment-assisted survival were calculated using SPSS software [19]. 


\section{RESULTS}

\section{IBMT confers a superior multilineage engraftment of AGM cells over IV administration.}

AGM cells contribute to low levels of chimerism when transplanted into adult recipients [16] due to their low HSC frequency/potential. Because IBMT constitutes a superior administration route than IV in the xenograft setting, we compared side-by-side the repopulation ability of murine E11.5 AGM cells after IBMT or IV transplantation. One ee of E11.5 AGM cells (CD45.2) were transplanted along with support cells (CD45.1) into adult lethally irradiated CD45.1 recipients by IV or IBMT (Fig. 1A). Analysis of PB chimerism at 16 weeks post-transplantation in reconstituted mice showed that IBMT-transplanted mice exhibited almost a 2 -fold higher level of donor reconstitution compared with IV-transplanted mice $(79 \pm 11 \%$ vs $46 \pm 12 \% ; P=0.1$, Fig. 1B). Regardless of the administration route, a donor multi-lineage (myeloid, B-cell and T-cell) engraftment was consistently observed (Fig. 1C). The increased engraftment observed in PB of IBMT-transplanted mice was accompanied by enhanced donor reconstitution in other hematopoietic tissues at 18 weeks post-transplantation (BM: $54 \pm 7 \%$ vs $28 \pm 10 \%$; spleen, $79 \pm 10 \%$ vs $49 \pm 17 \%$; thymus: $90 \pm 9 \%$ vs $47 \pm 20 \%$, IBMT vs IV, respectively. $P<0.05$ for pooled tissues, Fig. 1D).

The IBMT administration route did not skew the reconstitution pattern of the donor-engrafted cells since a similar percentage of myeloid $\left(\mathrm{Gr}-1^{+}\right), B\left(B 220^{+}\right)$and $\mathrm{T}\left(\mathrm{CD} 4^{+} / \mathrm{CD} 8^{+}\right)$cells was found within the CD45.2+ graft in BM, spleen and thymus (Fig. 2A,B). However, the percentage of donor cells within the Lineage-Sca- $1^{+} \mathrm{C}-\mathrm{kit}{ }^{+}$(LSK) compartment, which comprises the pool of immature stem and progenitor cells in the mouse hematopoietic system [20], was 50\%-60\% higher upon IBMT transplantation (Fig. 2C, left panel). Similarly, the percentages of CD45.2+ within the Sca-1+c-kit- and c-kit+Sca-1- compartments were higher in IBMT over IV-transplanted 
mice (Fig. 2C). Together, our data suggest that injection of AGM cells directly into the BM constitutes a more robust multilineage reconstitution approach than IV transplantation.

\section{IBMT confers higher hematopoietic reconstitution in secondary recipients and in limiting dilution experiments.}

To prove bona fide E11.5 AGM-derived HSC self-renewal and multi-lineage differentiation, primary IV and IBMT grafts (6 million unfractionated BM cells from 18-week primary recipients) were serially IV-transplanted into secondary recipients [21] (Fig. 3A) and hematopoietic reconstitution was assessed in the PB of secondary recipients at different time points after transplantation. Primary IBMT grafts resulted in consistently higher PB chimerism levels in secondary recipients as compared with primary IV grafts $(65 \%-90 \%$ vs $38 \%-52 \%, P<0.05$, Fig. 3B). Similarly higher multi-lineage donor chimerism was found after 20 weeks in PB, BM, spleen and thymus of secondary recipients transplanted with primary IBMT-engrafted cells as compared with primary IV grafts ( $\sim 90 \%$ vs $\sim 60 \%$. $P<0.05$ for pooled tissues, Fig. $3 \mathrm{C})$. The percentages of donor cells within the immature hematopoietic compartment (LSK), more primitive hematopoietic progenitors (Sca-1+c-kit-) or progenitor cells (c-kit+Sca-1-) was higher in IBMT over IV secondary recipients. (Fig. 3D). We then assessed whether IBMT administration facilitates the engraftment of lower doses of AGM cells (Fig. 3E). IBMT-transplanted 1 ee and 0.5 ee conferred $40 \%$ and $23 \%$, respectively, multilineage hematopoietic engraftment-assisted 20 -week survival. In contrast, equivalent AGM doses IV-transplanted conferred $25 \%$ and $7 \%$ engraftment, respectively, after 20 weeks (Fig. 3F). These results confirm that IBMT administration facilitates the read-out of hematopoietic reconstitution of embryonic tissues with very-low HSC content. 


\section{DISCUSSION}

Early in embryogenesis, hematopoietic stem and progenitor cells (HSPCs) such as those derived from yolk sac, AGM or ESCs, are very poorly represented due to either their scarcity or low developmental potential $[17,22]$. Definitive HSCs emerge from the AGM, and AGM-derived HSCs are the first cells reconstituting hematopoiesis in an irradiated adult animal [23]. Assessing the hematopoietic potential and plasticity of early AGM-derived cells relies on robust and sensitive in vivo long-term functional assays, and transplantation into irradiated mice is considered the in vivo gold standard test of HSC functionality. Although HSPC transplantation assays are commonly performed by IV administration, direct injection of cells into the BM is an alternative modality that has been used successfully to engraft human HSCs [5-7]. IBMT-based HSC delivery is, however, less common in the mouse syngeneic setting.

Here we compared the ability of IBMT and IV administration of AGM-derived cells to reconstitute the hematopoietic system of myeloablated recipients. In contrast to the IV route, IBMT administration resulted in significantly higher levels of AGM HSC long-term multilineage engraftment in all analyzed hematopoietic tissues of primary and secondary recipients. Importantly, the multilineage contribution pattern was not skewed in any hematopoietic organ by the IBMT administration route, suggesting that IBMT does not impair in vivo HSPC differentiation and migratory properties. However, IBMT conferred higher LSK long-term engraftment, in line with the superior IBMT reconstitution. Importantly, multilineage reconstitution was barely observed after 20 weeks when 0.5 ee were IV-transplanted, but was detected in $23 \%$ of the IBMT-transplanted mice receiving 0.5 ee. Our study indicates that IBMT represents a superior administration route to detect HSC activity for many applications, including when a limited number 
of HSCs are available from developmentally early sources such as the AGM, and when studying BM niche-HSC interactions [24]. We envision that the potential advantage of IBMT over IV might be: i) it bypasses the requirement for homing to the BM niche; ii) it facilitates survival within the niche; and iii) it prevents transplanted cells from being taken up by organs in vivo as occurs with intravenouslyinjected cells.

Recently, Daley and coworkers' demonstrated that recipient age influences the engraftment of embryonic HSCs [16] by showing that IV injection of AGM cells into neonatal recipients yielded a more robust engraftment than in adult recipients. Another study demonstrated that intrahepatic transplantation of human $\mathrm{CB}-\mathrm{CD} 34^{+}$cells into newborn immunodeficient mice provided enhanced hematopoietic engraftment without the requirement of accessory cells [25]. Our data suggest that IBMT transplantation of AGM cells in adult recipients may be used as an alternative modality to overcome the logistics of matching recipient's age with the nature of donor cells. The group of Ikehara have also compared IBMT and IV transplantation in mouse models. They showed that IBMT constitutes a powerful strategy in several scenarios, including treatment for autoimmune diseases [26]. Similarly, Li et al. analyzed the kinetics of hematopoietic reconstitution very early after IBMT or IV transplantation and found more donor-derived cells in IBMT-transplanted animals [14]. They also analyzed the long-term maintenance of donor-derived hematopoiesis after IBMT or IV using serial transplantation, and found that the frequency of donor-derived HSPCs was higher in IBMT- than in IV-transplanted tertiary recipients [13]. Additionally, Baba et al., used serial transplantation assays to demonstrate how IBMT accelerates donor cell engraftment and proliferation, and also facilitates reconstitution of dendritic cells [27]. In contrast to our results, de Haan's group showed that IBMT transplantation in a murine syngeneic setting does not provide enhanced engraftment in comparison with IV administration [15]. A likely explanation for this 
discrepancy may be the different ontogeny stage of donor cells since adult BM cells with a considerably higher HSC potential were used in the de Haan study.

Whether IBMT is superior to IV in terms of engraftment for other embryonic tissues, such as yolk sac, placenta, fetal liver or ESC-derived cells, remains unknown. Likewise, the engraftment potential of IBMT-transplanted ex vivo expanded AGM cells needs to be investigated in future studies. In the clinical setting, several trials have assessed the benefit of allogeneic transplantation using IV or IBMT administration [28]. One study observed reduced risk and severity of acute graft-versus-host disease in the IBMT cohort. Moreover, a 20-year follow-up trial concluded that intraosseus infusion of BM cells was a safe and efficient procedure [29]. Thus, we envision that IBMT will become the administration route of choice in experimental and clinical hematology.

ACKNOWLEDGMENTS AND FINANCIAL SUPPORT: This work was supported by the European Research Council CoG to P.M., the Spanish Ministry of Economy and Competitiveness (ISCIII/FEDER PI14/01119 to C.B and SAF2013-43065R to P.M), the Spanish Association Against Cancer to P.M. and C.B., the Fundación Inocente Inocente to PM, a Marie Curie Career Integration Grant to A.S-P (FP7-PEOPLE-2013-CIG-631171), and the Josep Carreras Dutch Leukemia Fund to P.M and ASP. C.B. is supported by a Miguel Servet II contract (CPII13/00011). C.P. is supported by PFIS scholarship (FI12/00468). P.M. also acknowledges the financial support from The Obra Social La Caixa-Fundació Josep Carreras and The Generalitat de Catalunya (SGR330).

CONFLICT OF INTEREST: The authors have no conflict of interest to disclose. All authors have read and approved the manuscript in its present form. 


\section{REFERENCES}

1. Orkin SH and LI Zon. (2008). Hematopoiesis: an evolving paradigm for stem cell biology. Cell 132:631-44.

2. Medvinsky A and E Dzierzak. (1996). Definitive hematopoiesis is autonomously initiated by the AGM region. Cell 86:897-906.

3. de Bruijn MF, NA Speck, MC Peeters and E Dzierzak. (2000). Definitive hematopoietic stem cells first develop within the major arterial regions of the mouse embryo. EMBO J 19:2465-74.

4. Rhodes KE, C Gekas, Y Wang, CT Lux, CS Francis, DN Chan, S Conway, SH Orkin, MC Yoder and HK Mikkola. (2008). The emergence of hematopoietic stem cells is initiated in the placental vasculature in the absence of circulation. Cell Stem Cell 2:252-63.

5. Levac K, P Menendez and M Bhatia. (2005). Intra-bone marrow transplantation facilitates pauciclonal human hematopoietic repopulation of NOD/SCID/beta2m(-/-) mice. Exp Hematol 33:141726.

6. Mazurier F, M Doedens, OI Gan and JE Dick. (2003). Rapid myeloerythroid repopulation after intrafemoral transplantation of NOD-SCID mice reveals a new class of human stem cells. Nat Med 9:959-63.

7. McKenzie JL, OI Gan, M Doedens and JE Dick. (2005). Human short-term repopulating stem cells are efficiently detected following intrafemoral transplantation into NOD/SCID recipients depleted of CD122+ cells. Blood 106:1259-61.

8. Montes R, V Ayllon, I Gutierrez-Aranda, I Prat, MC Hernandez-Lamas, L Ponce, S Bresolin, G Te Kronnie, M Greaves, C Bueno and P Menendez. (2011). Enforced expression of MLL-AF4 fusion in cord blood CD34+ cells enhances the hematopoietic repopulating cell function and clonogenic potential but is not sufficient to initiate leukemia. Blood 117:4746-58.

9. le Viseur C, M Hotfilder, S Bomken, K Wilson, S Rottgers, A Schrauder, A Rosemann, J Irving, RW Stam, LD Shultz, J Harbott, H Jurgens, M Schrappe, R Pieters and J Vormoor. (2008). In childhood acute lymphoblastic leukemia, blasts at different stages of immunophenotypic maturation have stem cell properties. Cancer Cell 14:47-58. 
10. Bardini M, PS Woll, L Corral, S Luc, L Wittmann, Z Ma, L Lo Nigro, G Basso, A Biondi, G Cazzaniga and SE Jacobsen. (2015). Clonal variegation and dynamic competition of leukemiainitiating cells in infant acute lymphoblastic leukemia with MLL rearrangement. Leukemia 29:3850.

11. Rodriguez R, R Rubio, M Masip, P Catalina, A Nieto, T de la Cueva, M Arriero, N San Martin, E de la Cueva, D Balomenos, P Menendez and J Garcia-Castro. (2009). Loss of p53 induces tumorigenesis in p21-deficient mesenchymal stem cells. Neoplasia 11:397-407.

12. Rubio R, A Abarrategi, J Garcia-Castro, L Martinez-Cruzado, C Suarez, J Tornin, L Santos, A Astudillo, I Colmenero, F Mulero, M Rosu-Myles, P Menendez and R Rodriguez. (2014). Bone environment is essential for osteosarcoma development from transformed mesenchymal stem cells. Stem Cells 32:1136-48.

13. Omae M, M Inaba, Y Sakaguchi, M Tsuda, T Miyake, J Fukui, H Iwai, T Yamashita and S Ikehara. (2008). Long-term maintenance of donor-derived hematopoiesis by intra-bone marrowbone marrow transplantation. Stem Cells Dev 17:291-302.

14. Li Q, H Hisha, R Yasumizu, TX Fan, GX Yang, Q Li, YZ Cui, XL Wang, CY Song, S Okazaki, T Mizokami, WH Cui, K Guo, M Li, W Feng, J Katou and S Ikehara. (2007). Analyses of very early hemopoietic regeneration after bone marrow transplantation: comparison of intravenous and intrabone marrow routes. Stem Cells 25:1186-94.

15. van Os R, A Ausema, B Dontje, M van Riezen, G van Dam and G de Haan. (2010). Engraftment of syngeneic bone marrow is not more efficient after intrafemoral transplantation than after traditional intravenous administration. Exp Hematol 38:1115-23.

16. Arora N, PL Wenzel, SL McKinney-Freeman, SJ Ross, PG Kim, SS Chou, M Yoshimoto, MC Yoder and GQ Daley. (2014). Effect of developmental stage of HSC and recipient on transplant outcomes. Dev Cell 29:621-8.

17. Kumaravelu P, L Hook, AM Morrison, J Ure, S Zhao, S Zuyev, J Ansell and A Medvinsky. (2002). Quantitative developmental anatomy of definitive haematopoietic stem cells/long-term 
repopulating units (HSC/RUs): role of the aorta-gonad-mesonephros (AGM) region and the yolk sac in colonisation of the mouse embryonic liver. Development 129:4891-9.

18. Bueno C, R Montes, T de la Cueva, I Gutierrez-Aranda and P Menendez. (2010). Intra-bone marrow transplantation of human CD34(+) cells into NOD/LtSz-scid IL-2rgamma(null) mice permits multilineage engraftment without previous irradiation. Cytotherapy 12:45-9.

19. Chillon MC, MT Gomez-Casares, CE Lopez-Jorge, C Rodriguez-Medina, A Molines, ME Sarasquete, M Alcoceba, JD Miguel, C Bueno, R Montes, F Ramos, JN Rodriguez, P Giraldo, M Ramirez, R Garcia-Delgado, JL Fuster, M Gonzalez-Diaz and P Menendez. (2012). Prognostic significance of FLT3 mutational status and expression levels in MLL-AF4+ and MLL-germline acute lymphoblastic leukemia. Leukemia 26:2360-6.

20. Okada S, H Nakauchi, K Nagayoshi, S Nishikawa, Y Miura and T Suda. (1992). In vivo and in vitro stem cell function of c-kit- and Sca-1-positive murine hematopoietic cells. Blood 80:3044-50.

21. Rundberg Nilsson A, CJ Pronk and D Bryder. (2015). Probing hematopoietic stem cell function using serial transplantation: Seeding characteristics and the impact of stem cell purification. Exp Hematol 43:812-817 e1.

22. Ayllon V, C Bueno, V Ramos-Mejia, O Navarro-Montero, C Prieto, PJ Real, T Romero, MJ Garcia-Leon, ML Toribio, A Bigas and P Menendez. (2015). The Notch ligand DLL4 specifically marks human hematoendothelial progenitors and regulates their hematopoietic fate. Leukemia 29:1741-53.

23. Sanchez MJ, A Holmes, C Miles and E Dzierzak. (1996). Characterization of the first definitive hematopoietic stem cells in the AGM and liver of the mouse embryo. Immunity 5:513-25.

24. Bueno C, LF Lopes, M Greaves and P Menendez. (2007). Toward development of a novel NOD/SCID-based in vivo strategy to model multiple myeloma pathogenesis. Exp Hematol 35:1477-8.

25. Navarro-Montero O, D Romero-Moya, R Montes, V Ramos-Mejia, C Bueno, PJ Real and P Menendez. (2012). Intrahepatic transplantation of cord blood CD34+ cells into newborn 
NOD/SCID-IL2Rgamma(null) mice allows efficient multi-organ and multi-lineage hematopoietic engraftment without accessory cells. Clin Immunol 145:89-91.

26. Kushida T, M Inaba, H Hisha, N Ichioka, T Esumi, R Ogawa, H lida and S Ikehara. (2001). Intrabone marrow injection of allogeneic bone marrow cells: a powerful new strategy for treatment of intractable autoimmune diseases in MRL/lpr mice. Blood 97:3292-9.

27. Baba S, M Inaba, H Iwai, M Taira, K Takada, H Hisha, T Yamashita and S Ikehara. (2005). Intrabone marrow-bone marrow transplantation facilitates hemopoietic recovery including dendritic cells. Immunobiology 210:33-42.

28. Ramirez PA, JE Wagner and CG Brunstein. (2010). Going straight to the point: intra-BM injection of hematopoietic progenitors. Bone Marrow Transplant 45:1127-33.

29. Hagglund H, M Remberger and O Ringden. (2014). Twenty-year follow-up of a randomized trial comparing intraosseous and i.v. BM transplantation. Bone Marrow Transplant 49:1541-2.

\section{FIGURE LEGENDS}


A

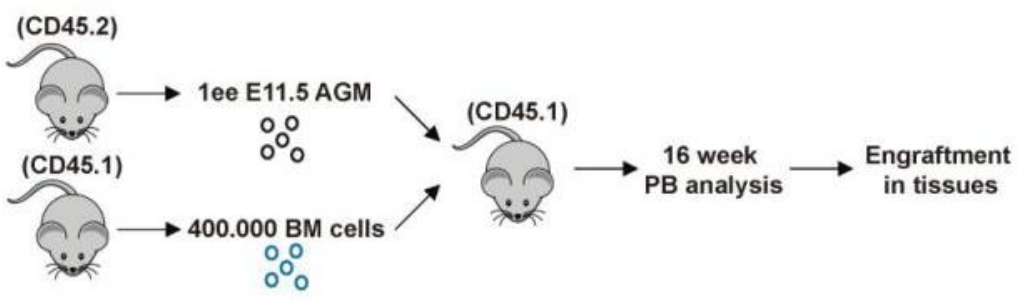

B
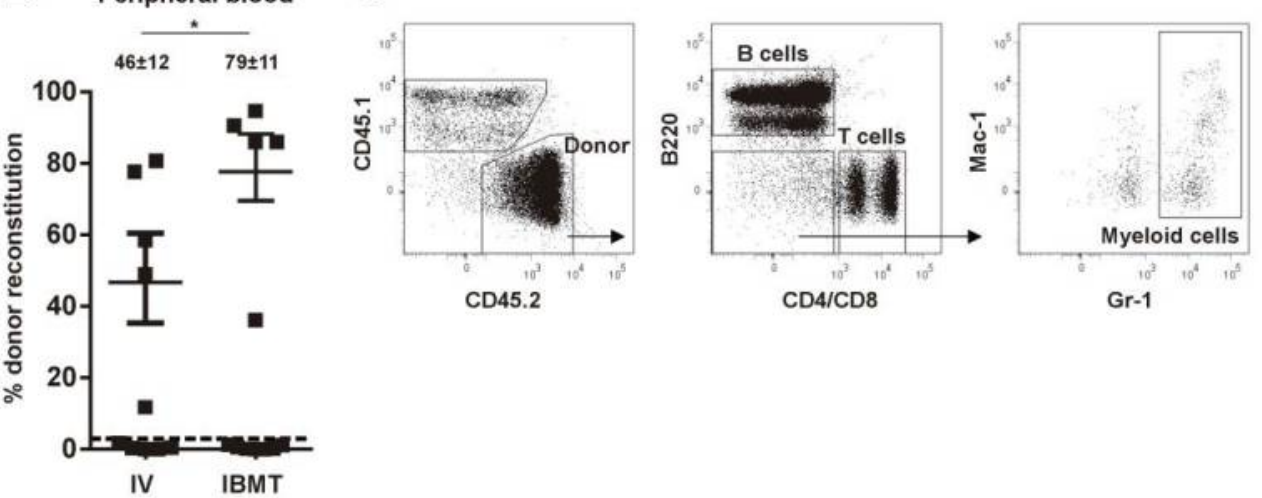

D

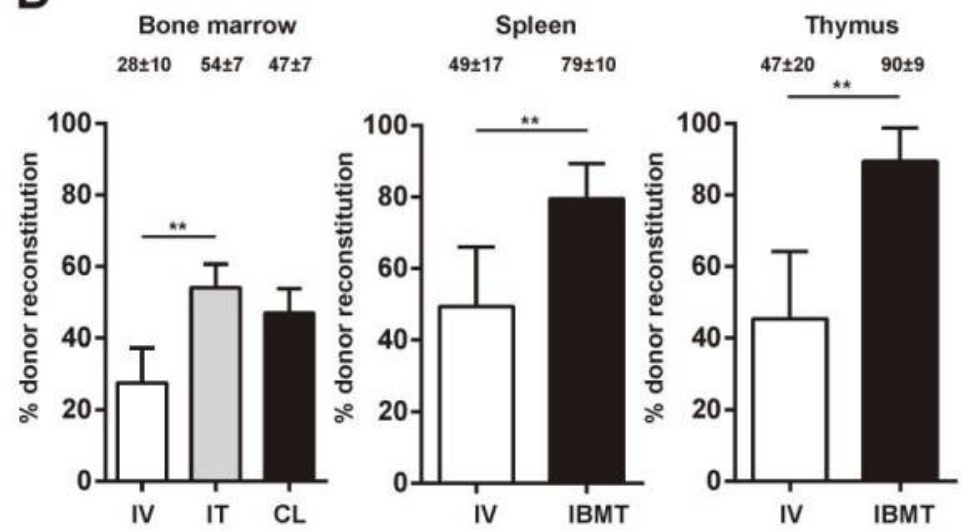

FIG. 1. IBMT confers a superior multilineage engraftment of AGM cells over IV administration. (A) Scheme depicting the experimental outline. Freshly isolated E11.5 AGM cells (CD45.2) were co-injected with support cells (CD45.1) either IV or IBMT into lethally 
irradiated recipients (CD45.1). Donor chimerism in PB was assessed at 16 weeks posttransplantation followed by tissue analysis after 18 weeks. (B) Mean percentage of donor chimerism in PB of mice transplanted with 1 ee AGM cells by IV $(n=12)$ or IBMT $(n=12)$ at 16 weeks. Mice were defined as long-term reconstituted if they had $\geq 1 \%$ donor contribution to the different lineages in PB at 16 weeks. (C) Representative FACS plots showing the gating strategy for donor chimerism and multi-lineage analysis in PB. (D) Mean percentage of donor chimerism in BM (IT: injected tibia; $C L$ : contralateral limb), spleen and thymus. Values show mean $\pm S E M$. ${ }^{*} P=0.1,{ }^{* *} P<0.05$. 
A
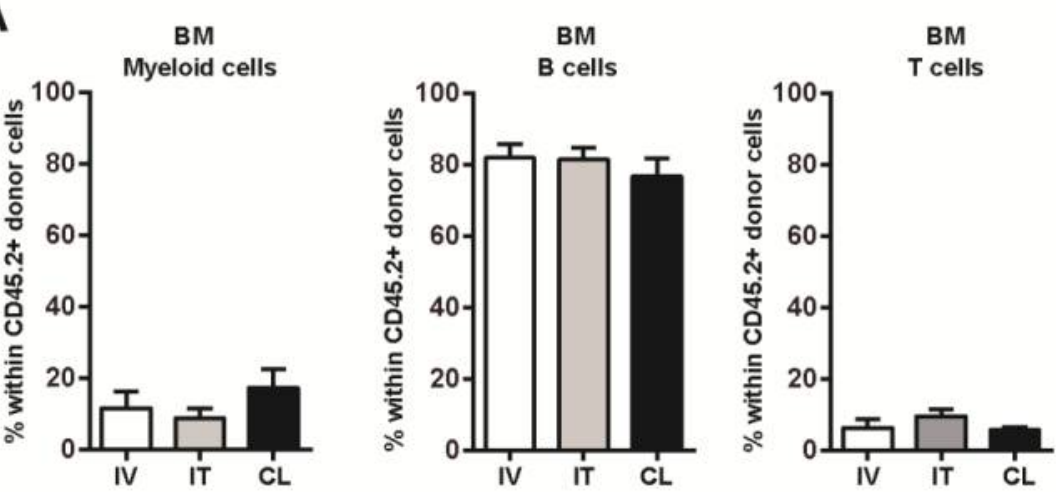

B
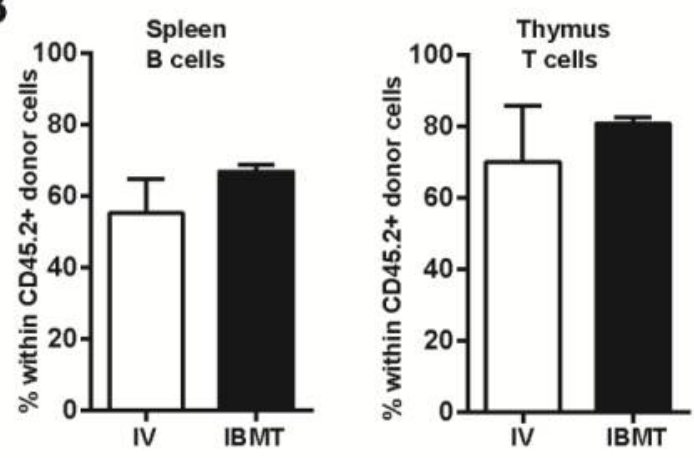

C
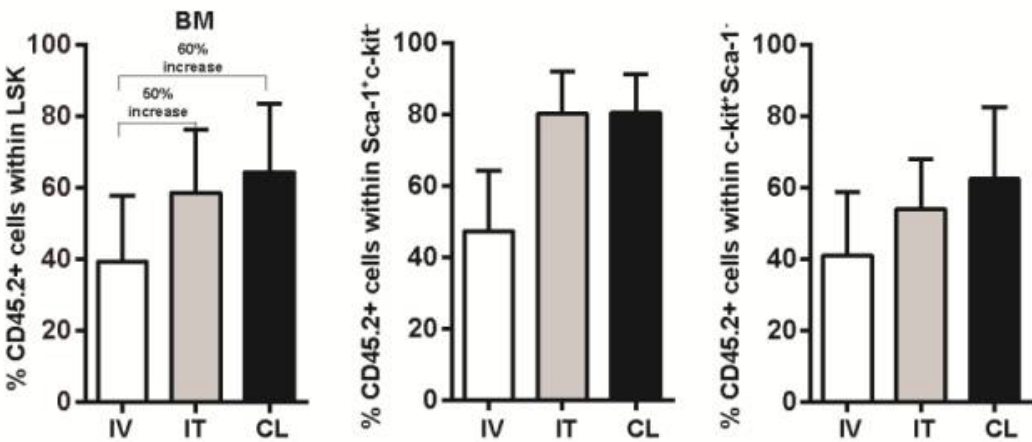

FIG. 2. Multilineage contribution pattern in different hematopoietic tissues is not skewed by the administration route. (A) Contribution of donor cells to B-cell $\left(B 220^{+}\right)$, myeloid $(\mathrm{Gr}-$ $\left.1^{+/} \mathrm{Mac}^{-1^{+}}\right)$and $\mathrm{T}$-cell $\left(\mathrm{CD}^{+} / \mathrm{CD}^{+}\right)$engraftment within BM (IT: injected tibia; CL: contralateral 
limb). (B) Contribution of donor cells to splenic B-cells (B220+) and double positive $\mathrm{CD} 4^{+} / \mathrm{CD} 8^{+}$ thymocytes in the spleen and thymus, respectively. Values show mean \pm SEM. (C) IBMT renders higher LSK (left panel), Sca-1+c-kit- (middle panel) and c-kit+Sca-1- (right panel) engraftment 18 weeks after transplantation. Values show mean \pm SEM. 
A

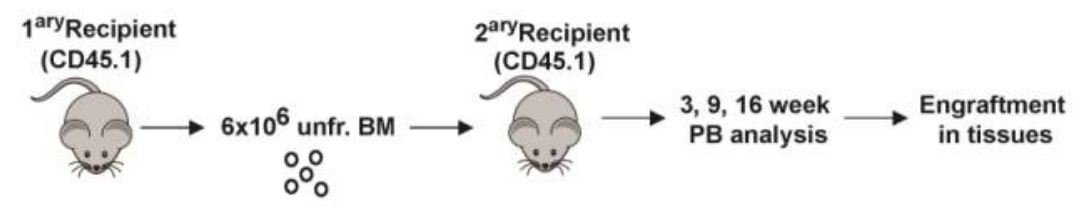

B

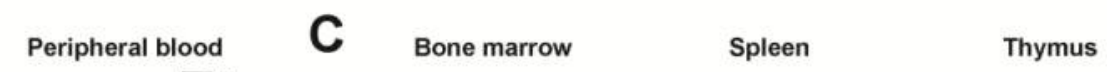
$\square_{\text {IвMT }}^{\text {IV }}$
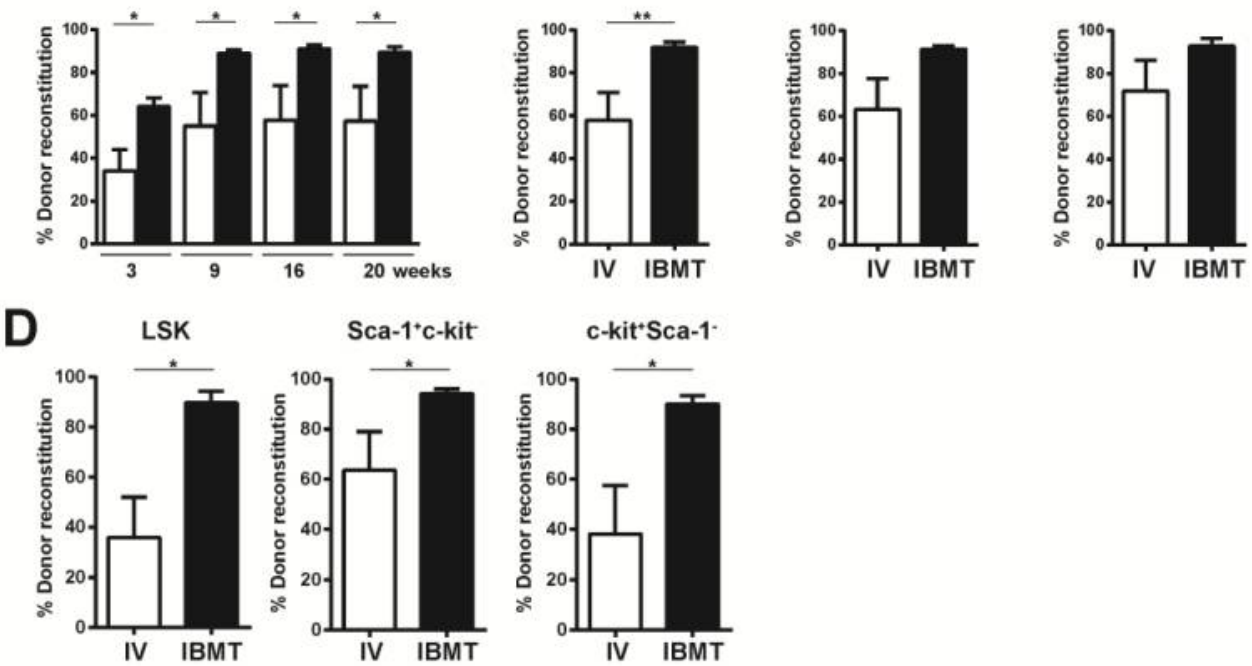

E
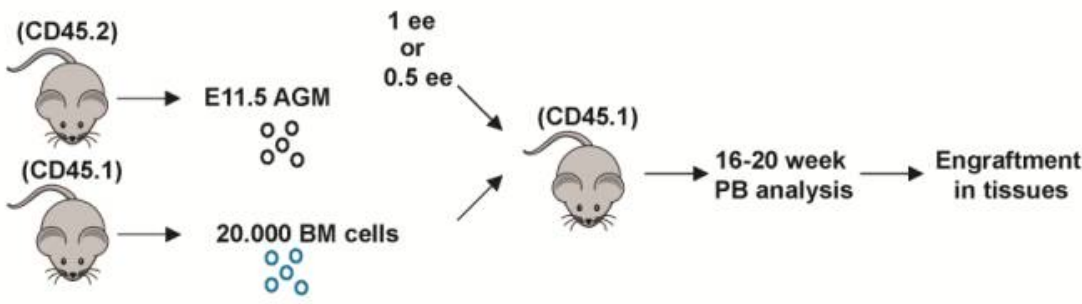

$\mathbf{F}$

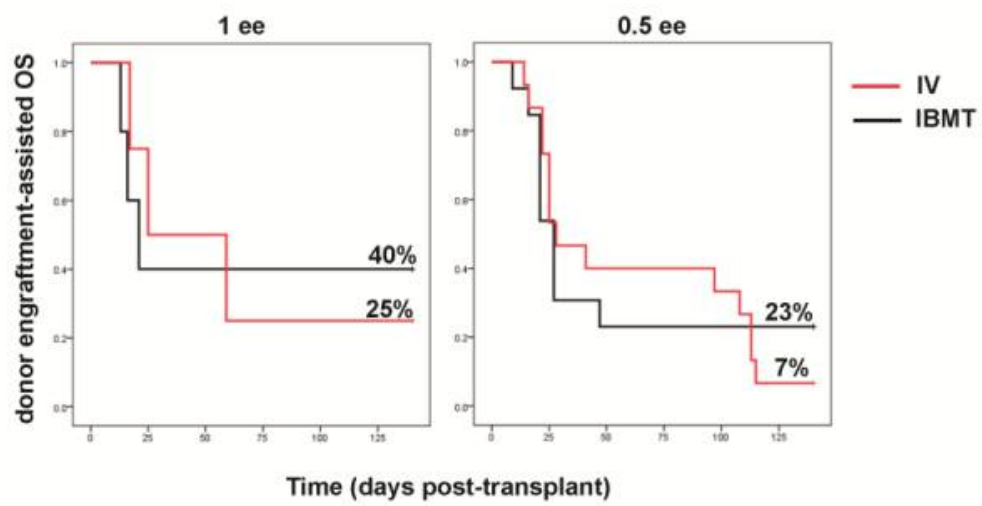

FIG. 3. IBMT confers higher donor-derived reconstitution in secondary recipients and in limiting dilution experiments. (A) Experimental outline for secondary transplantation. Six million BM cells from donor-reconstituted primary mice were IV re-transplanted into secondary lethally 
irradiated recipients ( $n=6$ IV and $n=6$ IBMT). (B) Donor chimerism in the PB of secondary recipients at the indicated time points. (C) Donor chimerism in BM, spleen and thymus of secondary recipients 20 weeks post-transplantation. (D) Percentage of donor (CD45.2+) cells within LSK (left panel), Sca-1+c-kit- (middle panel) and c-kit+Sca-1- (right panel) compartments in the BM of secondary recipients. (E) Experimental outline for limiting dilution experiments. One and 0.5 ee of fresh E11.5 AGM cells were injected along with support cells either IV or IBMT into lethally irradiated recipients. (F) Kaplan-Meier donor-assisted survival curves of mice upon IV or IBMT transplantation of 1 and 0.5 ee AGM cells. Mice from 5 independent experiments are included in the analysis. ${ }^{* *} P<0.05$.

Table S1. List of antibodies used in FACS experiments.

\begin{tabular}{|c|c|c|c|c|}
\hline Antigen & Fluorochrome & Clone & Dilution & Supplier \\
\hline B220 & PE-Cy7 & RA3-6B2 & $1 / 400$ & BD Bioscience \\
\hline B220 & VioBlue & RA3-6B2 & $1 / 400$ & Milteny Biotech \\
\hline B220 & PE-Cy5 & RA3-6B2 & $1 / 400$ & BioLegend \\
\hline CD4 & PE-Cy5 & GK1.5 & $1 / 800$ & BioLegend \\
\hline CD4 & PE & GK1.5 & $1 / 200$ & Milteny Biotech \\
\hline CD45.1 & APC & A20 & $1 / 200$ & BD Bioscience \\
\hline CD45.2 & APC-Cy7 & 104 & $1 / 200$ & BD Bioscience \\
\hline CD5 & PE-Cy5 & $53-7.3$ & $1 / 400$ & BioLegend \\
\hline CD8a & PE-Cy5 & $53-6.7$ & $1 / 800$ & BioLegend \\
\hline CD8a & FITC & $53-6.7$ & $1 / 400$ & Milteny Biotech \\
\hline CD8a & PE & $53-6.7$ & $1 / 400$ & Milteny Biotech \\
\hline C-kit & PE & $2 B 8$ & $1 / 200$ & BD Bioscience \\
\hline Gr-1 & PE-Cy5 & RB6-8C5 & $1 / 800$ & BioLegend \\
\hline Mac-1 & PE-Cy5 & M1/70 & $1 / 800$ & BioLegend \\
\hline Mac-1 & V450 & M1/70 & $1 / 400$ & BD Bioscience \\
\hline Sca-1 & FITC & E13-161.7 & $1 / 200$ & BD Bioscience \\
\hline Ter119 & PE-Cy5 & TER119 & $1 / 200$ & BioLegend \\
\hline
\end{tabular}

